\title{
Etoposide, doxorubicin and cisplatin plus mitotane in the treatment of advanced adrenocortical carcinoma: a large prospective phase II trial
}

\author{
Alfredo Berruti, Massimo Terzolo ${ }^{1}$, Paola Sperone, Anna Pia², \\ Silvia Della Casa ${ }^{3}$, David J Gross', Carlo Carnaghi, ${ }^{5}$, Paolo Casali ${ }^{6}$, \\ Francesco Porpiglia ${ }^{7}$, Franco Mantero ${ }^{8}$, Giuseppe Reimondo ${ }^{1}$, \\ Alberto Angeli ${ }^{1}$ and Luigi Dogliotti
}

\author{
Oncologia Medica and ${ }^{1}$ Medicina Interna, Università di Torino, Azienda Ospedaliera San Luigi, Regione Gonzole \\ 1010043 Orbassano, Torino, Italy \\ ${ }^{2}$ Endocrinologia, Azienda Ospedaliera Santa Croce e Carle, Cuneo, Italy \\ ${ }^{3}$ Istituto di Endocrinologia, Università Cattolica, Policlinico A Gemelli, Roma, Italy \\ ${ }^{4}$ Endocrinology and Metabolism Service, Division of Medicine, Hadassah University Hospital, Jerusalem, Israel \\ ${ }^{5}$ Oncologia Medica, Istituto Clinico Humanitas, Rozzano, Milano, Italy \\ ${ }^{6}$ Oncologia Medica, Istituto Nazionale dei Tumori, Milano, Italy \\ ${ }^{7}$ Urologia, Azienda Ospedaliera San Luigi, Orbassano, TO, Italy \\ ${ }^{8}$ Dipartimento di Scienze Mediche Chirurgiche, Endocrinologia, Università di Padova, Italy
}

(Requests for offprints should be addressed to Luigi Dogliotti; Email: luigi.dogliotti@unito.it)

\begin{abstract}
To investigate the activity of etoposide, doxorubicin, and cisplatin plus mitotane in the management of advanced adrenocortical carcinoma (ACC) patients, 72 patients with measurable disease not amenable to radical surgery were enrolled in a prospective, multicenter phase II trial. EDP schedule (etoposide $100 \mathrm{mg} / \mathrm{m}^{2}$ on days $5-7$, doxorubicin $20 \mathrm{mg} / \mathrm{m}^{2}$ on days 1 and 8 , and cisplatin $40 \mathrm{mg} / \mathrm{m}^{2}$ on days 1 and 9) was administered intravenously every 4 weeks. Concomitantly, patients were given up to $4 \mathrm{~g} /$ day of oral mitotane. Five patients achieved a complete response and $30 \mathrm{a}$ partial response, for an overall response rate of $48.6 \%(95 \% \mathrm{Cl}: 37.1-60.3)$. Median time to progression in responding patients was 18 months. The EDP regimen was well tolerated, leukopenia being the dose limiting toxicity. One toxic related death due to septic shock, however, was registered. Radical surgical resection of residual disease after chemotherapy was performed in 10 patients. The overall survival of patients attaining a disease free status (clinical complete responders + radically resected) was significantly higher than that of patients with partial response or no response $(P<0.002)$. Androgen secretion was associated with long survival, while glucocorticoid secretion was associated with poor prognosis both in univariate and multivariate analysis. In conclusion, EDP plus mitotane is an active and manageable combination scheme for ACC patients. Surgical resection of residual disease subsequent to chemotherapy leads to a more favourable outcome. The natural history of the disease is significantly influenced by the secretory status of the tumor.
\end{abstract}

Endocrine-Related Cancer (2005) 12 657-666

\section{Introduction}

Adrenocortical carcinoma (ACC) is an extremely rare disease with an annual incidence ranging between 0.5 and 2 cases per million population (Crucitti et al. 1996,
Icard et al. 2001) in western countries. ACC patients often present with clinical syndromes secondary to the production of corticosteroids, although some patients bear non-functioning neoplasms (Allolio et al. 2004). The stage of the disease at diagnosis and complete 
surgical resection are recognized as the strongest predictors of long-term survival (Allolio et al. 2004). In two large multicenter European series, it was found that approximately $20 \%$ of patients have metastatic disease at the time of presentation (Crucitti et al. 1996, Icard et al. 2001), one out of four patients is left with residual disease after operation and half of those who had an apparently radical resection eventually relapse, often with distant metastases (Crucitti et al. 1996, Icard et al. 2001). The prognosis of patients with nonoperable disease is dismal with an estimated 5 year survival rate close to 0\% (Crucitti et al. 1996, Icard et al. 2001). The treatment options for advanced ACC are limited. The adrenolytic drug mitotane $(1,1$ dichloro-2 (o-chlorophenyl)-2-(p-chloro-phenyl) etane) has been widely employed but it is difficult to appraise its efficacy from retrospective studies which have seldom used standard criteria to assess objective responses (Luton et al. 1990). Transient partial responses were found in 19 to $34 \%$ of cases, whereas complete responses were anecdotal (Wooten \& King 1993, Haak et al. 1994, Kasperlik-Zaluska 2000, Terzolo et al. 2000, Baudin et al. 2001). The toxicity of mitotane is admittedly a major limitation to its use. There is limited experience with cytotoxic therapy in ACC. Only eight small phase II trials have been published up to now (Van Slooten \& van Oosterom 1983, Decker et al. 1991, Schlumberger et al. 1991, Bukowski et al. 1993, Bonacci et al. 1998, Khan et al. 2000, Williamson et al. 2000, Abraham et al. 2002). They enrolled a limited number of patients with unresectable disease and only three of them had enough power to assess a cytotoxic activity (at least 30 patients enrolled) (Bukowski et al. 1993, Williamson et al. 2000, Abraham et al. 2002). Cisplatin is the cytotoxic agent most frequently used in this disease. Cisplatin in combination with mitotane provided a response rate close to $30 \%$ (Bukowski et al. 1993). The combination of cisplatin with other cytotoxic agents such as etoposide, doxorubicin, and 5-fluorouracil did not improve this rate (Van Slooten \& van Oosterom 1983, Schlumberger et al. 1991, Bonacci et al. 1998, Williamson et al. 2000). In ACC, the multidrugresistance gene (MDR-1) is highly expressed and high levels of p-glycoprotein (PgP) are expected (Villa et al. 1999). PgP acts as a drug efflux pump and is a major cause of chemotherapy failure in cancer treatment. Chemotherapy activity of ACC could be conceivably improved by combining cytotoxic drugs with a modulator of MDR-1 gene expression (Villa et al. 1999). Mitotane is able to interfere with $\mathrm{PgP}$ thus facilitating intracellular drug accumulation and enhancing drug cytotoxicity (Bates et al. 1991, Villa et al.
1999). Since 1993, a multicenter phase II trial started in Italy with the aim of testing the activity of mitotane administered in association to EDP (Etoposide, Doxorubicin and Cisplatin) in advanced ACC patients. Preliminary data have been published in 1998 (Berruti et al. 1998). This paper presents the final results.

\section{Patients and methods}

Patients with histologically confirmed adrenocortical carcinoma and metastatic or locally advanced disease not amenable to radical surgery were considered eligible. Other inclusion criteria were: age less than 65 years, measurable disease according to WHO criteria (Miller et al. 1981), clinical and radiologic evidence of disease progression, life expectancy of over three months, performance status (PS) $<3$ (according to ECOG scale), adequate bone marrow reserve (white blood cells $>3500 / \mathrm{mm}^{3}$ or neutrophil $>1500 / \mathrm{mm}^{3}$ and platelets $>150.000 / \mathrm{mm}^{3}$ ), adequate renal and hepatic function (creatinine clearance $>60 \mathrm{ml} / \mathrm{min}$, bilirubin, aspartate aminotransferase and alanine aminotransferase $<1.5$ times the upper limit of normality), absence of previous chemotherapy treatment, apart from mitotane received in adjuvant setting, no history of radiation therapy to measurable lesions, written informed consent according to the ethic committee's requirements. Exclusion criteria included: presence of brain metastases, history of other neoplasms, history of myocardial infarction or unstable angina, cardiac insufficiency, uncontrolled arrythmia, presence of active infection, presence of osteoblastic bone metastasis as the only site of disease.

\section{Treatment plan}

The EDP scheme consisted of intravenous administration of etoposide at doses of $100 \mathrm{mg} / \mathrm{m}^{2}$ on days $5-7$, doxorubicin $20 \mathrm{mg} / \mathrm{m}^{2}$ on days 1 and 8 , cisplatin $40 \mathrm{mg} / \mathrm{m}^{2}$ on days 2 and 9 . Cycles were repeated every 4 weeks. Treatment was delayed for 1 week in case of neutrophil count of 1000 to $1500 / \mathrm{mm}^{3}$, platelet count of 50000 to $100000 / \mathrm{mm}^{3}$, or grade $>2$ extrahematologic toxicity on day of treatment. Dose reduction $(25 \%)$ was planned in case of neutrophil count $<500 /$ $\mathrm{mm}^{3}$ and/or platelet count $<30000 / \mathrm{mm}^{3}$, experienced in the interval (nadir) between two cycles. Mitotane was given orally at the starting dose of $1 \mathrm{~g}$ /day, with further progressive dose increments up to $4 \mathrm{~g}$ /day or the maximum tolerated dose. Mitotane was administered concomitantly with chemotherapy, during the rest period between successive cycles, and afterwards until disease progression or the onset of severe toxicity. 
All patients received concomitant adrenal replacement therapy to prevent adrenal insufficiency. EDP administration was planned for a maximum of six cycles. Treatment was discontinued early in case of unacceptable toxicity, a patient's refusal to continue treatment or disease progression.

\section{Patient evaluation}

Pre-chemotherapy staging included: physical examination, assessment of PS, routine chemistry (including blood cell counts, electrolytes, renal and liver function tests), determination of steroid hormone levels, electrocardiography and echocardiography and computed tomography (CT) scanning of chest and abdomen. Physical examination and routine chemistry were repeated before each cycle of chemotherapy. Steroids were measured in-house at each participating center using radioimmunoassay methods and commercially available reagents. All samples for an individual subject were determined in a single assay in duplicate. Intra- and inter-assay coefficients of variation for all hormone variables were less than 10 and $15 \%$ respectively.

\section{Evaluation of response and toxicity}

Tumor status and endocrine evaluation were assessed at 3 and 6 months during chemotherapy administration, and every 3 months afterwards until evidence of disease progression. Objective responses were classified according to WHO criteria (Miller et al. 1981): complete response (CR) was defined as the disappearance of all clinical evidence of tumor on physical examination or on radiography; partial response (PR) required at least a $50 \%$ decrease in all measurable tumor size for at least 4 weeks; stable disease was defined as a $<50 \%$ regression of all measurable lesions or $<25 \%$ increase in the size of any measurable lesion for at least 4 weeks; progressive disease (PR) was defined as a $>25 \%$ increase in the size of any measurable lesion or appearance of new lesions; complete hormone response was defined as the normalization of all adrenocortical steroids; and partial hormone response was defined as a reduction greater than $50 \%$ of pretreatment levels. Changes in hormone levels per se were not considered as a response to treatment. Conversely, patients attaining a complete clinical response with persistence of abnormal hormone levels were considered as partial responders. Toxicities were graded using WHO criteria (Miller et al. 1981). Time to progression (TTP) was defined as the time from the beginning of treatment until date of disease progression and overall survival (OS) was defined as the time from beginning of treatment until date of death or the last date of follow-up.

\section{Statistical methods}

The primary end point of the study was the assessment of the response rate. According to the optimal twostage phase II study design by Simon (1989), the treatment program was designed to refuse response rates $<20 \%$ (p0) and to provide a statistical power of $80 \%$ in assessing the activity of the regimen as a $35 \%$ response rate. The upper limit for first-stage drug rejection was 5 responses of the first 22 patients, the upper limit of second-stage rejection was 19 responses of 72 cases consecutively enrolled. Secondary end points were the determination of time to progression and overall survival. All activity and survival analyses were performed including all registered patients, according to an "intent to treat" principle. All survival functions were computed using the Kaplan Meier method and compared using the log-rank test. A landmark method (Simon 1989) was used to determine the survival from a point 4 months after the initiation of therapy to provide an unbiased conditional estimate of TTP and OS. A period of 4 months was selected because this was the time point of first treatment evaluation in most cases. To investigate the relative importance of various factors for TTP and OS, the Cox proportional hazard model was applied. The Statistica (Statsoft, Tulsa, OK, USA) for Windows package was used for statistical computation.

\section{Results}

\section{Patient characteristics}

The study was open for enrollment between 1993 and 2003, during this period 72 eligible patients were enrolled from 26 institutions. Table 1 shows the pretreatment patient characteristics. One patient did not meet the eligibility criteria since he was 73 years old in accordance. According to the intent to treat analysis by which he was evaluated. In 61 patients $(84.7 \%)$ primary disease had been previously surgically removed, 19 patients $(26.3 \%)$ had undergone multiple resections for local recurrence or metastatic disease. The median time between diagnosis and initiation of EDP+mitotane treatment was 7.3 months (range 0-252 month).

An endocrine work up including at least measurement of serum and urinary cortisol, one androgen steroid (17-hydroxyprogesterone, dehydroepiandrosterone sulphate) as well as testosterone in the female and estradiol in the male population, was available in 
Table 1 Patient Characteristics

\begin{tabular}{|c|c|}
\hline Characteristics & No. (\%) of patients \\
\hline N. & 72 \\
\hline \multicolumn{2}{|l|}{ Age } \\
\hline median (range) & $50(18-73)$ \\
\hline Males & $24(33.3 \%)$ \\
\hline Females & $48(66.7 \%)$ \\
\hline \multicolumn{2}{|l|}{ Performance Status (ECOG) } \\
\hline 0 & $31(43.0 \%)$ \\
\hline 1 & $28(38.9 \%)$ \\
\hline 2 & $11(15.3 \%)$ \\
\hline 3 & $2(2.8 \%)$ \\
\hline \multicolumn{2}{|l|}{ Stage at diagnosis } \\
\hline I & $1(1.4 \%)$ \\
\hline II & $24(33.3 \%)$ \\
\hline III & $30(41.7 \%)$ \\
\hline IV & $17(23.6 \%)$ \\
\hline \multicolumn{2}{|l|}{ Stage at the study entry } \\
\hline Locally advanced & $9(12.5 \%)$ \\
\hline Metastatic & $63(87.5 \%)$ \\
\hline \multicolumn{2}{|l|}{ Diesease sites } \\
\hline Adrenal & $39(54.2 \%)$ \\
\hline Lung & $36(50.0 \%)$ \\
\hline Liver & $18(25.0 \%)$ \\
\hline Skin/lymphnodes & $10(13.9 \%)$ \\
\hline Abdomen & $16(22.2 \%)$ \\
\hline Bone & $3(4.2 \%)$ \\
\hline \multicolumn{2}{|l|}{ Number of disease sites } \\
\hline 1 & $37(51.4 \%)$ \\
\hline 2 & $24(33.4 \%)$ \\
\hline 3 & $6(8.3 \%)$ \\
\hline 4 & $5(6.9 \%)$ \\
\hline \multicolumn{2}{|l|}{ Number of previous surgeries } \\
\hline 0 & $11(15.3 \%)$ \\
\hline 1 & $42(58.3 \%)$ \\
\hline 2 & $14(19.4 \%)$ \\
\hline 3 & $5(6.9 \%)$ \\
\hline Mitotane as adjuvant treatment & $13(18.0 \%)$ \\
\hline \multicolumn{2}{|l|}{ Symptoms/signs } \\
\hline Hypertension & $23 / 70(32.8 \%)$ \\
\hline Menstrual disorders ${ }^{\star *}$ & $14 / 25(56.0 \%)$ \\
\hline Hirsutism* & $21 / 48(43.7 \%)$ \\
\hline Acne & $15 / 70(21.4 \%)$ \\
\hline Obesity & $17 / 70(24.3 \%)$ \\
\hline Moon facies & $14 / 70(20.0 \%)$ \\
\hline Buffalo hump & $8 / 70(11.4 \%)$ \\
\hline Palpable abdominal mass & $7 / 70(10.0 \%)$ \\
\hline Gynecomastia*** & $3 / 24(12.5 \%)$ \\
\hline Diabetes mellitus & $7 / 70(10.0 \%)$ \\
\hline Hypokaliemia & $8 / 70(11.4 \%)$ \\
\hline Strie rubrae & $5 / 70(7.1 \%)$ \\
\hline Muscle weakness & $6 / 70(8.6 \%)$ \\
\hline Virilization* & $6 / 48(12.5 \%)$ \\
\hline \multicolumn{2}{|l|}{ Type of hypersecretion } \\
\hline No secretion & $22 / 70(31.4 \%)$ \\
\hline Androgens alone & $14 / 70(20.0 \%)$ \\
\hline Cortisol alone & $10 / 70(14.3 \%)$ \\
\hline Cortisol + Estradiol & $3 / 70(4.3 \%)$ \\
\hline
\end{tabular}

Table 1 continued

\section{Characteristics}

\section{Aldosterone alone}

Androgens + Cortisol

Aldosterone + Cortisol

Aldosterone + Androgens

Aldosterone + Androgens + Cortisol

*only female patients were included.

${ }^{* *}$ only female patients in premenopausal status were evaluated.

${ }^{* * *}$ only male patients were included.

70 patients. In 48 of them $(68.6 \%)$ there was evidence of hormone hypersecretion that was clinically overt in 29 patients $(41.4 \%)$.

\section{Treatment activity}

An objective regression was observed in 35 out of 72 patients $(48.6 \%$; 95\% CI: 37.1-60.3\%). Five patients $(6.9 \%)$ achieved a clinical complete response, 20 $(27.8 \%)$ stable disease, while $16(22.2 \%)$ progressed. No difference in response rate has been observed according to functional and non functional tumors. Forty-two patients out of 48 showing hormonal hypersecretion at baseline had hormones monitored during treatment. Hormonal response was observed in 28 cases $(66.7 \%), 16(38.1 \%)$ attained a complete hormonal regression. Eight patients showed no change in hormone levels during treatment while in six patients hormone levels increased remarkably. Surgery of residual disease was performed in five patients who were classified as partial responders and in six patients who showed a tumor shrinkage $<50 \%$ (classified as stable disease). Ten of these patients underwent radical removal of the disease and became disease free $(13.8 \%)$. In one patient no neoplastic cells were observed at residual histology. Residual disease in radically resected patients was primary adrenal cancer or local recurrence in seven patients, lung metastases in two patients, and primary adrenal cancer + liver metastases in one patient. Surgery was also attempted as a salvage option in three patients with progressive disease after chemotherapy, but radical resection was never obtained.

\section{Time to progression and overall survival}

At the last follow-up appointment (March 2004), 56 patients $(77.8 \%)$ had disease progression and 43 $(59.7 \%)$ had died. Median TTP and OS of the entire cohort were 9.1 and 28.5 months respectively (18.2 and 47.7 months respectively, in the patient subset attaining a disease response). TTP was greater than 2 years in 14 patients and greater than 5 years in seven 


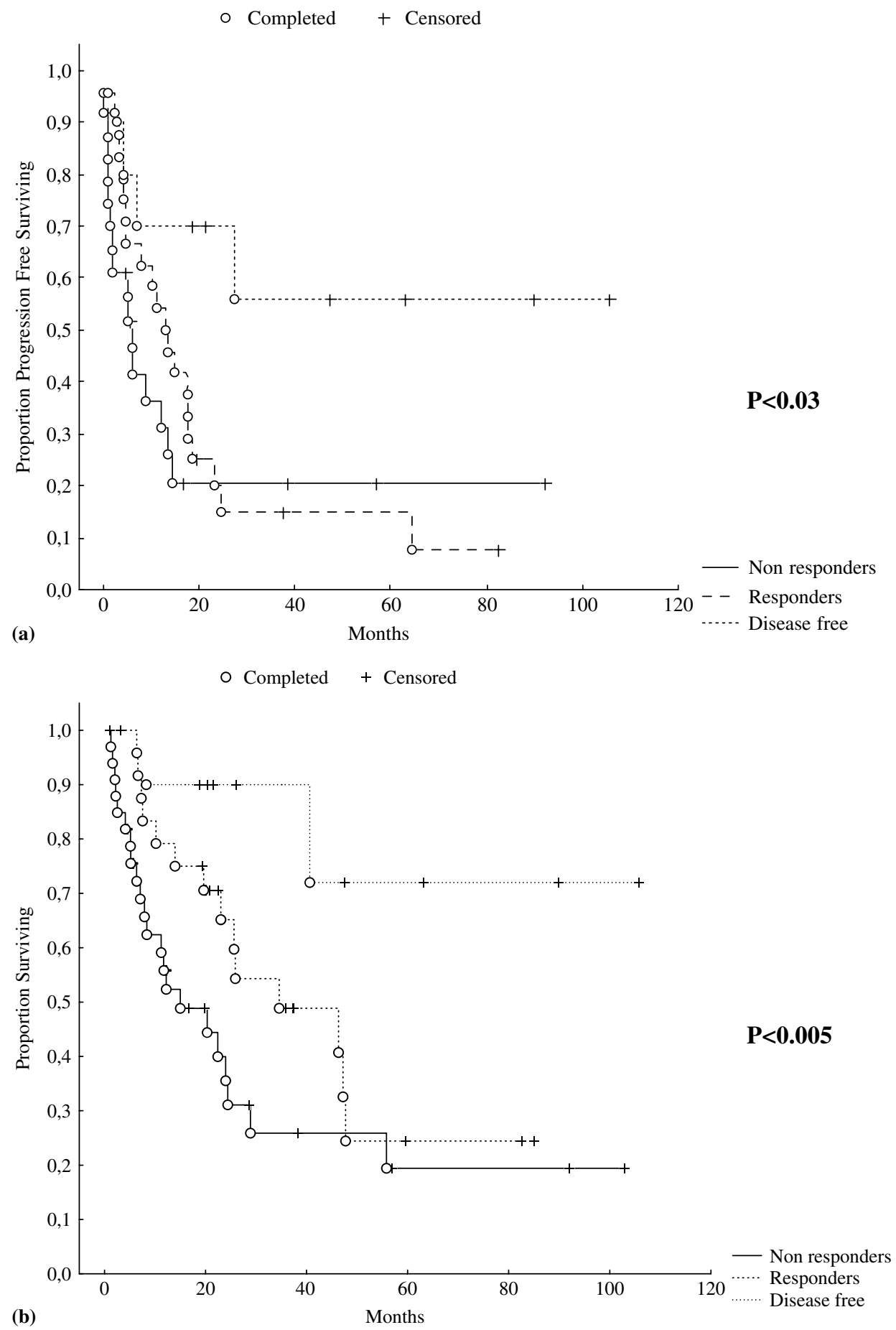

Figure 1 Time to progression (a) and overall survival (b) after a landmark point of 4 months in patients attaining a disease free status vs patients with partial response, and patients not responding.

patients. OS was greater than 2 years in 35 patients and greater than 5 years in 10 patients. Using the landmark method, patients who attained a disease free status after treatment, i.e. complete responders (as total of 5) plus those radically operated (a total of 10), showed a longer TTP and OS as compared with patients showing a partial response and those with no response (Fig. 1). A number of baseline prognostic 
Table 2 Predictive factors for time to progression and overall survival according to an univariate analysis

\begin{tabular}{|c|c|c|c|c|}
\hline & Time of progression & $\boldsymbol{P}$ & Survial & $\boldsymbol{P}$ \\
\hline \multicolumn{5}{|l|}{ Sex } \\
\hline Males & 8.2 (3.7-12.8) & \multirow[t]{2}{*}{$=0.61$} & $23.6(9.2-38.1)$ & $=0.27$ \\
\hline Females & $11.1(5.8-16.4)$ & & $38.6(18.6-58.6)$ & $=0.03$ \\
\hline \multicolumn{5}{|l|}{ Disease Free Survival } \\
\hline$\leq 2$ years & $8.6(4.6-12.6)$ & \multirow[t]{2}{*}{$=0.32$} & $26.4(15.9-37.0)$ & \multirow[t]{2}{*}{$=0.03$} \\
\hline$>2$ years & $18.3(3.6-33.0)$ & & $51.7(10.0-93.4)$ & \\
\hline \multicolumn{5}{|l|}{ Performance Status } \\
\hline 0 & $12.0(0.4-13.6)$ & \multirow{3}{*}{$=0.18$} & $44.6(14.2-75.1)$ & \multirow{3}{*}{$=0.01$} \\
\hline 1 & $11.1(48-17.4)$ & & $32.9(23.3-42.5)$ & \\
\hline $2-3$ & $5.1(0.6-9.6)$ & & $11.1(7.4-14.8)$ & \\
\hline \multicolumn{5}{|l|}{ No sites of disease } \\
\hline 1 & $13.1(4.8-21.3)$ & \multirow[t]{2}{*}{$=0.14$} & $44.6(15.8-73.4)$ & \multirow[t]{2}{*}{$<0.01$} \\
\hline$>1$ & $8.2(5.0-11.4)$ & & $15.2(0.0-31.0)$ & \\
\hline \multicolumn{5}{|l|}{ Functional status } \\
\hline No secretion & $21.5(5.4-37.5)$ & \multirow{3}{*}{$=0.03$} & 35.1 (20.4-68.9) & \multirow{3}{*}{$=0.01$} \\
\hline $\begin{array}{l}\text { Cortisol } \pm \text { Androgens } \\
\text { or Estradiol }\end{array}$ & $8.2(5.7-10.8)$ & & $16.2(3.4-29.1)$ & \\
\hline Androgens & $17.6(15.1-20.1)$ & & $60.4(44.3-100.0)$ & \\
\hline
\end{tabular}

Data are median months (95\% Confidence Intervals).

factors such as sex, disease free survival (DFS), PS, single vs multiple sites of disease, and type of hormonal secretion, have been tested as predictors of both TTP and OS. In univariate analysis (Table 2), short disease free survival, low PS, multiple disease sites and the functional status of the tumor were significantly related to poor survival. Only the functional status of the tumor significantly correlated to TTP. Patients with androgen hypersecretion showed longer OS, while patients with cortisol hypersecretion (with or without androgen hypersecretion) showed shorter OS, as compared to their counterparts. Factors attaining a statistical significance in univariate analysis were

Table 3 Independent prognostic factors

\begin{tabular}{lccc}
\hline & \multicolumn{3}{c}{ Overall survival } \\
\cline { 2 - 4 } & $\begin{array}{c}\text { Hazard } \\
\text { Risk }\end{array}$ & $\begin{array}{c}\text { 95\% } \\
\text { Coefficient } \\
\text { Interval }\end{array}$ & $\boldsymbol{P}$ \\
\hline $\begin{array}{c}\text { DFS } \\
\quad \leqslant 2 \text { y vz }>2 y)\end{array}$ & 0.300 & $0.124-0.729$ & $<0.02$ \\
$\begin{array}{l}\text { Multiple disease sites } \\
\text { vz 1 site }\end{array}$ & 1.767 & $0.907-3.443$ & $=0.09$ \\
$\begin{array}{l}\text { PS } \\
(0 \text { vz 1 vz 2-3) }\end{array}$ & 1.228 & $0.800-1.885$ & $=0.35$ \\
$\begin{array}{l}\text { Functional status } \\
\text { (Cortisol } \pm \text { androgens } \\
\text { vz non functioning } \\
\text { vz androgen) }\end{array}$ & 0.641 & $0.422-0.973$ & $<0.04$ \\
\hline
\end{tabular}

evaluated in multivariate analysis (Table 3). DFS and the functional status of the tumor were independent variables associated to OS while the presence of multiple sites of disease and patient PS did not enter the model.

\section{Treatment administered and toxicity}

A total of 357 EDP courses were administered (median 6, range 1-13). Associated side effects are reported in Table 4 . The most frequent toxicity was hematologic: grade $>2$ toxicity for white blood cells were recorded in $10 \%$ of patients at recycle and in $33 \%$ of patients at nadir. There was a toxic death due to septic shock caused by prolonged neutropenia. The most common extra-hematologic side effects were gastrointestinal, neurologic, asthenia and myalgias, but were less than grade 3 in most cases. Neurologic toxicity was central in two patients, peripheral in 14 cases, and was both central and peripheral in five cases. All patients received at least one treatment cycle and $38(52.8 \%)$ patients completed the treatment plan (six cycles), including three patients who received 8,8 , and 13 cycles respectively. The EDP regimen was delayed 1 week for 30 patients $(41.7 \%)$, and 2 weeks for 14 patients $(19.4 \%)$. Leukopenia and infections were the principal reasons for these delays. Dose reduction of all the three EDP drugs was performed in seven patients due to hematologic toxicity. The planned dose of mitotane ( $4 \mathrm{~g}$ daily) was regularly received by 21 patients 
Table 4 Treatment Toxicity

\begin{tabular}{|c|c|c|c|c|c|}
\hline WHO & 0 & 1 & 2 & 3 & 4 \\
\hline \multicolumn{6}{|l|}{ Gastrointestinal } \\
\hline Nausea/vomiting & 7 (10.0\%) & $21(30.0 \%)$ & 34 (48.6\%) & 8 (11.4\%) & - \\
\hline Mucositis & $50(71.4 \%)$ & $10(14.2 \%)$ & $7(10.0 \%)$ & $3(4.3 \%)$ & - \\
\hline Diarrhea & $53(75.7 \%)$ & $8(11.4 \%)$ & $7(10.0 \%)$ & $1(1.4 \%)$ & $1(1.4 \%)$ \\
\hline Hepatic & $60(85.7 \%)$ & $5(7.2 \%)$ & $3(4.3 \%)$ & $2(2.8 \%)$ & - \\
\hline \multicolumn{6}{|l|}{ Hematologic } \\
\hline \multicolumn{6}{|l|}{ At recycle: } \\
\hline WBC & 33 (47.1\%) & 13 (18.6\%) & 17 (24.3\%) & $6(8.6 \%)$ & $1(1.4 \%)$ \\
\hline Neutrophil & $33(47.1 \%)$ & $7(10.0 \%)$ & $20(28.5 \%)$ & $6(8.6 \%)$ & $4(5.7 \%)$ \\
\hline Platelets & 62 (88.6\%) & $3(4.3 \%)$ & $5(7.1 \%)$ & - & - \\
\hline Hemoglobin & 22 (31.4\%) & 22 (31.4\%) & $23(32.8 \%)$ & $3(4.3 \%)$ & - \\
\hline \multicolumn{6}{|l|}{${ }^{\star}$ At nadir: } \\
\hline WBC & $15(23.4 \%)$ & 7 (10.9\%) & $21(32.8 \%)$ & $18(28.1 \%)$ & $3(4.7 \%)$ \\
\hline Neutrophil & $15(23.4 \%)$ & $2(3.1 \%)$ & $16(25.0 \%)$ & $18(28.1 \%)$ & $13(20.3 \%)$ \\
\hline Platelets & $48(75.0 \%)$ & $6(9.4 \%)$ & $5(7.8 \%)$ & $2(3.1 \%)$ & $3(4.7 \%)$ \\
\hline \multicolumn{6}{|l|}{ Others toxicities } \\
\hline Renal & $61(87.1 \%)$ & $7(10.0 \%)$ & 2 (2.8\%) & - & - \\
\hline Astenia & $21(30.0 \%)$ & $16(22.8 \%)$ & $29(41.4 \%)$ & $4(5.7 \%)$ & - \\
\hline Myalgias & $55(78.5 \%)$ & $12(17.1 \%)$ & $2(2.8 \%)$ & $1(1.4 \%)$ & - \\
\hline Cardiac & 69 (98.6\%) & - & - & $1(1.4 \%)$ & - \\
\hline Neurologic & $59(84.3 \%)$ & $13(18.6 \%)$ & 7 (10.0\%) & $1(1.4 \%)$ & - \\
\hline
\end{tabular}

*Data available on 64 patients.

$(29.2 \%)$. The maximum tolerated dose was $3 \mathrm{~g} /$ day for 35 patients $(48.6 \%), 2 \mathrm{~g} /$ day for ten $(13.9 \%)$, and $1 \mathrm{~g} /$ day for five $(6.9 \%)$. One patient received no mitotane at all because he had experienced severe peripheral neurotoxicity while taking the drug in adjuvant setting. The main reasons for reducing mitotane doses were neurologic or gastrointestinal toxicities. Five patients interrupted mitotane before ending the treatment plan due to neurologic toxicity (two patients), gastrointestinal toxicity (two patients) and PS deterioration (one patient).

\section{Discussion}

The rarity of ACC has encumbered the prospects for a widely accepted first-choice treatment for advanced disease. The association of etoposide, doxorubicin, and cisplatin was first developed by Preusser et al. (1989) in the treatment of patients with gastric cancer. These authors reported high activity but also greater toxicity. With respect to the original EDP scheme, the dose of etoposide was reduced from $120 \mathrm{mg} / \mathrm{m}^{2}$ to $100 \mathrm{mg} / \mathrm{m}^{2}$ while the doses of cisplatin and adriamycin did not change. The feasibility of the EDP scheme in association with mitotane was tested by our group in patients with advanced ACC (Berruti et al. 1992, Pia et al. 1995). The present study has shown that EDP plus mitotane is an active regimen confirming our preliminary observations (Berruti et al. 1998). About half of patients did respond and most patients had hormonal hypersecretion controlled. To our knowledge this is the largest prospective study reported to date. Even with cooperation of several oncological, endocrinological and surgical centers, it took 10 years to accrue the planned number of patients. The therapeutic activity of EDP plus mitotane seems to be higher both in terms of response rate and survival than that of either mitotane alone or other chemotherapy schemes, with or without mitotane (Van Slooten \& van Oosterom 1983, Decker et al. 1991, Schlumberger et al. 1991, Bukowski et al. 1993, Bonacci et al. 1998, Khan et al. 2000, Williamson et al. 2000, Abraham et al. 2002) There may be several explanations. The combination of mitotane could have yielded complementary cytotoxic effects or have hampered resistance mechanisms. Alternatively, since this chemotherapeutic regimen encompasses all drugs that as single agents or in combination were found to be active, the overall activity could be the result of additive effects. Although the majority of responses were partial, they were durable with a median TTP in responding cases of 18 months. The median survival of 28 months in the whole series is noteworthy when considering that $87 \%$ patients had metastatic disease. The five year survival rate of $15 \%$ 
compares favourably with that reported in two large series (Crucitti et al. 1996, Icard et al. 2001). The prospective nature is a strength of the present study while the absence of a control group is a limitation. It should be noted, however, that in this rare neoplasm there is not a single randomized clinical trial conducted up to now worldwide. ACC is a heterogeneous disease with occasional patients surviving more than 10 years despite metastatic disease. This observation points to the importance of searching for prognostic factors. We showed in this study that common prognostic factors in solid tumors such as DFI, patient PS, overall tumor load significantly correlated with survival in univariate analysis but only DFI showed an independent prognostic role in multivariate analysis. Noteworthy, both in univariate and multivariate analysis, tumors secreting androgens or precursors alone had a better prognosis than tumors secreting cortisol alone or with androgens. These data are in agreement with previous reports (Ribeiro et al. 2000, Icard et al. 2001) and are biologically plausible. In fact, while androgen excess may exert anti-catabolic effects and stimulate erythropoiesis, hypercortisolism has been advocated to be an adverse prognostic factor in advanced small cell lung cancer (Dimopoulos et al. 1992). Moreover, the functional status in our series was the only variable predictive for TTP. Since TTP is a measure of treatment efficacy, the presence of hypercortisolism could also negatively influence the probability to obtain a durable response to our regimen. Our main strategy was to obtain a complete remission of the disease, which was obtained in five cases, and to convert these clearly unresectable tumors to resectable ones, which was accomplished in ten patients. From a landmark of four months after the initiation of therapy, the survival of patients who became disease free after chemotherapy alone, or after chemotherapy followed by adjunctive surgery was significantly greater than that of the remainders. These data confirm the importance of radical surgery in favoring long term survival of advanced patients (Schulick \& Brennan 1999). As a whole the EDP plus mitotane regimen was manageable. The most severe and dose limiting toxicity was leukopenia leading to fatal septic shock in one case. As previously observed (Berruti et al. 1998), the addition of mitotane to the EDP regimen accounted for additive neurologic toxicity. Other treatment related toxicities seen during this study were without clinical significance. To conclude, the results of this large prospective phase II study showed that EDP plus mitotane is an active and manageable scheme in the treatment of advanced ACC. Surgical resection of residual disease appears to play a pivotal role in leading to a more favourable outcome. Since the functional status of the tumor is predictive of both treatment efficacy and patient prognosis, the measurement of steroid hormones at baseline should be considered as part of the management of patients with ACC.

\section{Acknowledgements}

This study was supported by the Associazione Italiana per la Ricerca Contro il Cancro (AIRC), Milan, Italy. We would like to thank the following principal investigators and participating institutions for their contribution: Dr M Boscaro (Endocrinologia, Università di Ancona, Ancona, Italy); Dr O Daniele (Divisione di Oncologia Medica, Azienda Ospedaliera, Padova, Italy); Dr P Loli (Divisione di Endocrinologia, Ospedale Niguarda Cà Granda, Milano, Italy); Prof A De Matteis (Oncologia-Endocrinologia Istituto Tumori, Fondazione Pascale, Napoli, Italy); Dr C Verusio (Ambulatorio di Radiochemioterapia, Istituto San Raffaele del Monte Tabor, Milano, Italy); Dr D Bisbocci (Divisione di Medicina Interna, Università di Torino, Ospedale San Vito, Torino, Italy); Dr C Marcocci (Istituto di Endocrinologia, Metodologia Clinica e Medicina del Lavoro, Università di Pisa, Tirrenia, Pisa, Italy); Prof B Ambrosi (Istituto di Scienze Endocrine, Università di Milano, Ospedale Maggiore, Milano, Italy); Dr F Desiderio (Divisione di Oncologia, Ospedale di Rimini, Italy); Dr A (Frassoldati, Cattedra di Oncologia, Università di Modena, Italy); Dr G Moro (Divisione di Radioterapia, Ospedale degli Infermi, Biella, Italy); Dr M Zini (Servizio di Endocrinologia, Arcispedale Santa Maria Nuova, Reggio Emilia, Italy); Dr D Amadori (Unità Operativa di Oncologia, Presidio Ospedaliero 'GB Morgagni-L. Pierantoni' Forlì, Italy); Dr C Cianti (Oncologia Medica, Ospedale S. Spirito in Sassia, Roma, Italy); Dr L Laudadio (Unità Operativa di Oncologia Medica, Presidio Ospedaliero Renzetti, Lanciano, Perugia, Italy); Prof G Biasco (Cattedra di Oncologia Medica, Policlinico Sant'Orsola, Bologna, Italy); Dr M Marangolo (Divisione di Oncologia Medica, Ospedale Santa Maria delle Croci, Ravenna, Italy); Dr F Roila (Divisione di Oncologia, Policlinico, Perugia, Italy); Dr A Savarese (Oncologia Medica, Istituto Regina Elena, Roma, Italy); Dr U Folco (Servizio di Oncologia, Ospedale Santa Corona, Pietra Ligure, Savona, Italy); Dr K Engstrom (Norwegian Radium Hospital, Oslo, Norway).

The authors declare that there are no conflicts of interest that would prejudice this study's impartiality. 


\section{References}

Abraham J, Bakke S, Rutt A, Meadows B, Merino M, Alexander R, Schrump D, Bartlett D, Choyke P, Robey R et al. 2002 A Phase II trial of combination chemotherapy and surgical resection for the treatment of metastatic adrenocortical carcinoma: continuous infusion doxorubicin, vincristine, and etoposide with daily mitotane as a P-glycoprotein antagonist. Cancer 94 2333-2343.

Allolio B, Hahner S, Weismann D \& Fassnacht M 2004 Management of adrenocortical carcinoma. Clinical Endocrinology 60 273-287.

Bates SE, Shieh CY, Mickley LA, Dichek HL, Gazdar A, Loriaux DL \& Fojo AT 1991 Mitotane enhances cytotoxicity of chemotherapy in cell lines expressing a multidrug resistance gene (mdr-1/P-glycoprotein) which is also expressed by adrenocortical carcinomas. Journal of Clinical Endocrinology and Metabolism 73 18-29.

Baudin E, Pellegriti G, Bonnay M, Penfornis A, Laplanche A, Vassal G \& Schlumberger M 2001 Impact of monitoring plasma 1,1-dichlorodiphenildichloroetane (o,p'DDD) levels on the treatment of patients with adrenocortical carcinoma. Cancer 92 1385-1392.

Berruti A, Terzolo M, Paccotti P, Veglio F, Pia A, Dogliotti L \& Angeli A 1992 Favourable response of metastatic adrenocortical carcinoma to etoposide, adriamycin, and cisplatin chemotherapy. Report of two cases. Tumori $\mathbf{7 8}$ 345-348.

Berruti A, Terzolo M, Pia A, Angeli A \& Dogliotti L 1998 Mitotane associated with etoposide doxorubicin and cisplatin in the treatment of advanced adrenocortical carcinoma. Cancer 83 2194-2200.

Bonacci R, Gigliotti A, Baudin E, Wion-Barbot N, Emy P, Bonnay M, Cailleux AF, Nakib I \& Schlumberger M 1998 Cytotoxic therapy with etoposide and cisplatin in advanced adrenocortical carcinoma. British Journal of Cancer 78 546-549.

Bukowski RM, Wolfe M \& Levine HS 1993 Phase II trial of mitotane and cisplatin in patients with adrenal carcinoma. A Southwest Oncology Group Study. Journal of Clinical Oncology 11 161-165.

Crucitti F, Bellantone R, Ferrante A, Boscherini M \& Crucitti P 1996 The Italian Registry for adrenal cortical carcinoma: analysis of a multi-institutional series of 129 patients. The ACC Italian Registry Study Group. Surgery 119 161-170.

Decker RA, Elson P, Hogan TF, Citrin DL, Westring DW, Banerjee TK, Gilchrist KW \& Horton J 1991 Eastern Cooperative Oncology Group study 1879: mitotane and adriamycin in patients with advanced adrenocortical carcinoma. Surgery 110 1006-1013.

Dimopoulos MA, Fernandez JF, Samaan NA, Holoye PY \& Vassilopoulou-Sellin R 1992 Paraneoplastic Cushing Syndrome as an adverse prognostic factor in patients who die early with small-cell lung cancer. Cancer $6966-71$.
Haak HR, Hermans J \& van de Velde CJ 1994 Optimal treatment of adrenocortical carcinoma with mitotane: results in a consecutive series of 96 patients. British Journal of Cancer 69 947-951.

Icard P, Goudet P, Charpenay C, Andreassian B, Carnaille B, Chapuis Y, Cougard P, Henry JF \& Proye C 2001 Adrenocortical carcinomas: surgical trends and results of a 253-patient series from the French Association of Endocrine Surgeons Study Group. World Journal of Surgery 25 891-897.

Kasperlik-Zaluska AA 2000 Clinical results of the use of mitotane for adrenocortical carcinoma. Brazilian Journal of Medical and Biological Research 33 1191-1196.

Khan TS, Imam H, Juhlin C, Skogseid B, Grondal S, Tibblin S, Wilander E, Oberg K \& Eriksson B 2000 Streptozotocin and o,p'DDD in the treatment of adrenocortical cancer patients: long term survival in its adjucvant use. Annals of Oncology 11 1281-1287.

Luton JP, Cerdas S, Billaud L, Thomas G, Guilhaume B, Bertagna X, Laudat MH, Louvel A, Chapuis Y, Blondeau P et al. 1990 Clinical features of adrenocortical carcinoma, prognostic factors, and the effect of mitotane therapy. New England Journal of Medicine 322 1195-1201.

Miller AB, Hoogstraten B, Staquet M \& Winkler A et al. 1981 Reporting results of cancer treatment. Cancer 47 207-214.

Pia A, Berruti A, Terzolo M, Paccotti P, Letizia C, Dogliotti L \& Angeli A 1995 Feasibility of the association of mitotane with etoposide, adriamycin, and cisplatin combination chemotherapy in advanced adrenocortical cancer patients. Annals of Oncology 6 509-510.

Preusser P, Wilke H, Acterrath W, Fink U, Lenaz L, Heincke A, Meyer J \& Meyer Buente J 1989 Phase II study with the combination of etoposide, doxorubicin and cisplatin in advanced measurable gastric cancer. Journal of Clinical Oncology 171 1310-1317.

Ribeiro RC, Michalkiewicz EL, Figueiredo BC, DeLacerda L, Sandrini F, Pianovsky MD, Sampaio G \& Sandrini R 2000 Adrenocortical tumors in children. Brazilian Journal of Medical and Biological Research 33 1225-1234.

Schlumberger M, Brugieres L, Gicquel C, Travagli JP, Droz JP \& Parmentier C 1991 5-fluorouracil, doxorubicin, and cisplatin as treatment for adrenal cortical carcinoma. Cancer 67 2997-3000.

Schulick RD \& Brennan MF 1999 Long-term survival after complete resection and repeat resection in patients with adrenocortical carcinoma. Annals of Surgical Oncology 6 719-726.

Simon R 1989 Optimal two-stage designs for phase II clinical trials. Controlled Clinical Trials 10 1-10.

Terzolo M, Pia A, Berruti A, Osella G, Ali A, Carbone V, Testa E, Dogliotti L \& Angeli A 2000 Low-dose monitored mitotane treatment achieves the therapeutic range with manageable side effects in patients with adrenocortical cancer. Journal of Clinical Endocrinology and Metabolism 85 2234-2238. 
Van Slooten H \& van Oosterom AT 1983 CAP

(Cyclophosphamide, Doxorubicin and Cisplatin) regimen in adrenal cortical carcinoma. Cancer Treatment Reports 67 377-379.

Villa R, Orlandi L, Berruti A, Dogliotti L \& Zaffaroni N 1999 Modulation of cytotoxic drug activity by mitotane and lonidamine in human adrenocortical carcinoma cells. International Journal of Oncology 14 133-138.
Williamson SK, Lew D, Miller GJ, Balcerzak SP, Baker LH \& Crawford ED 2000 Phase II evaluation of cisplatin and etoposide followed by mitotane at disease progression in patients with locally advanced or metastatic adrenanocortical carcinoma: a Southwest Oncology Group Study. Cancer 88 1159-1165.

Wooten MD \& King DK 1993 Adrenocortical carcinoma. Epidemiology and treatment with mitotane and a review of the literature. Cancer 72 3145-3155. 